\title{
Prognostic value of tumor mutation burden (TMB) and INDEL burden (IDB) in cancer: current view and clinical applications
}

\author{
Anwaar Saeed ${ }^{1}$, Mohamed E. Salem ${ }^{2}$ \\ ${ }^{1}$ Department of Medicine, Division of Medical Oncology, Gastrointestinal Oncology Program, Kansas University Cancer Center, Kansas City, KS, \\ USA $;{ }^{2}$ Department of solid tumor oncology, Levine Cancer Institute, Charlotte, NC, USA \\ Correspondence to: Anwaar Saeed, MD. Department of Medicine, Division of Medical Oncology, Gastrointestinal Oncology Program, Kansas \\ University Cancer Center, 2330 Shawnee Mission Parkway, Suite 210, Westwood Administrative Building, Westwood, Kansas City, KS 66205, USA. \\ Email: asaeed@kumc.edu. \\ Provenance and Peer Review: This article was commissioned by the editorial office, Annals of Translational Medicine. The article did not undergo \\ external peer review. \\ Comment on: Wu HX, Wang ZX, Zhao Q, et al. Tumor mutational and indel burden: a systematic pan-cancer evaluation as prognostic biomarkers. \\ Ann Transl Med 2019;7:640.
}

Submitted Apr 02, 2020. Accepted for publication Apr 15, 2020.

doi: 10.21037/atm-2020-75

View this article at: http://dx.doi.org/10.21037/atm-2020-75

Tumor specific mutations have the potential capability of generating diverse repertoire of immunogenic nonself peptides "neoantigens" that can be recognized by T-cell receptors in the context of MHC complex, leading to the generation of anti-tumor immune response (1). The burden of such neoantigens has been linked to the underlying total amount of nonsynonymous mutation per mega base of DNA also called tumor mutational burden (TMB) $(2,3)$. The neoantigen/TMB is an emerging biomarker for the efficacy and clinical response to immune checkpoint inhibitors (ICIs) (4). While the majority of the tumor somatic sequence mutations are non-synonymous single nucleotide variants (SNVs) (5), insertion and deletion (INDEL) mutations occur less frequently but have a superior potential of generating immunogenic peptides. These mutations lead to an open reading frame as a result of genomic Insertions or deletions of base pairs, generating a unique sequence from the germline counterpart (6).

While TMB has been studied as a predictive biomarker to guide immunotherapy, the general prognostic impact has not been studied on a wide scale in solid tumors. In this context, this published article by Wu et al. looked at the general prognostic impact of TMB in addition to INDEL burden (IDB) across 20 solid tumor subtypes in 6,035 patients. The clinical and survival data for these patients were obtained from The Cancer Genome Atlas (TCGA) and the curated somatic mutation data for each tumor were extracted from the Multi-Center Mutation Calling in Multiple Cancers (MC3) project. The median TMB ranges from 0.34 to $13.09 \mathrm{mut} / \mathrm{Mb}$. Survival analysis revealed three trends in which TMB impacted overall survival. The TMB worse group, in which high TMB was associated with worse prognosis in comparison to low TMB group. This group includes eight cancer types, adrenocortical carcinoma, cholangiocarcinoma, colon adenocarcinoma, esophageal carcinoma, renal clear cell carcinoma, hepatocellular carcinoma, mesothelioma and pancreatic adenocarcinoma. The TMB better group, in which high TMB predicted better prognosis includes bladder urothelial carcinoma, renal papillary cell carcinoma, stomach adenocarcinoma, endocervical adenocarcinoma, ovarian serous cystadenocarcinoma and uterine corpus endometrial carcinoma. The TMB similar group, in which the TMB did not have any significant impact on the overall survival, includes head and neck squamous cell carcinoma, lung adenocarcinoma, lung squamous cell carcinoma, cutaneous melanoma, uveal melanoma and uterine carcinosarcoma (7).

This is the first and largest systematic study of kind dissecting the prognostic impact of TMB in addition to IDB across 20 solid tumor types. These findings are pivotal to our understanding of the TMB as it relates to the intrinsic biology of different solid tumors reflecting on its impact on overall survival. The three trends of TMB impact on survival observed by $\mathrm{Wu}$ et al., shed light on the essential 
role that TMB might play in risk stratification in addition to therapy selection in the current scheme of management of different tumor types. This is especially true for TMBworse prognostic group as the high TMB might not be predictive for response to immunotherapeutic agents as its critical for risk stratification.

IDB impact on survival along with TMB in this study highlighted key information where IDB assessment is critical. The study observed that in the TMB similar group, IDB was able to skew the overall survival in two tumor subtypes. High IDB was significantly associated with worse prognosis in uterine carcinosarcoma, while high IDB in cutaneous melanoma was a predictor for a better prognosis (7). In these instances, IDB is supplementary in assessing overall survival. If similar findings are validated in a future study, IDB should also be considered in future risk stratification in those tumor subtypes.

The TMB and IDB prognostic impact reported by $\mathrm{Wu}$ et al. were similar to that reported in the literature for few cancer types but varied for the majority. This is not unexpected, as different studies have used different cut points to assign the TMB high category (3). In this study, the cut-point to determine high TMB was assigned based on the point associated with the highest statistical significance in a range from the $50^{\text {th }}$ to $90^{\text {th }}$ percentiles in each cancer subtype. The observed trend of TMB impact on survival in non-small cell lung cancer (NSCLC) varied across different studies. While Wu et al. reported no significant impact of TMB on overall survival, another study evaluated TMB from 908 specimens of early stage NSCLC [the lung adjuvant cisplatin evaluation (LACE)-Bio-II study]. High TMB (>8 mutations/ $\mathrm{Mb}$ ) was associated with favorable overall survival, disease-free survival, and lung cancer specific survival (8). Goodman et al. have also reported that high TMB in NSCLC patients was associated with a better progression-free survival than that with low-TMB group (9). These results need further validation on larger scale, tumor specific study taking into account other confounding factors in order to assess the true impact of TMB on survival in NSCLC. An example of those potential biases is the variation of volume of various pathologic disease stages in published studies. While considering that pathologic staging is a major prognostic factor in most cancer types, lumping stage I-III disease in one survival analysis would likely have an impact on study outcome. Another potential play factor is the variation between studies in the burden of various pathogenic molecular alterations that have an independent prognostic impact on overall survival.
The TMB prognostic impact findings by $\mathrm{Wu}$ et al. have further ramifications for tumors intrinsic biologic properties. Two different tumor subtypes with high TMB might behave differently. An example of how high TMB might reflect the underlying tumor biologic behavior is in high grade serous ovarian cancer. Per Wu et al., these tumors fall in the TMB better prognostic category and thus tumors with high TMB showed better survival outcomes in comparison to low TMB counterpart. This finding can be explained based on the fact that tumors enriched in mutations might harbor more tumor specific neoantigens, which stimulate T-cell specific immune response, lymphocyte infiltration and thus up regulation of immune checkpoints. This hypothesis is supported by Strickland et al. findings that high grade serous ovarian cancer with BRCA1/2 mutation harbor more predicted neoantigens in comparison to homologous recombination (HR) proficient tumors (tumors with low mutation burden). The higher predicted neoantigen burden in these tumors was also associated with a better overall survival in addition to enrichment in immune signatures associated with tumor cytotoxicity. These tumors also displayed higher infiltrating lymphocytes with higher expression of PD-1 and PD-L1 (10).

On the other hand, some tumors with high TMB might be enriched in mutations associated with aggressive behavior or therapy resistance including immunotherapy. In such instances TMB high status might not be well predictive of response to therapy but rather a predictive biomarker for risk stratification. Clear cell renal cell carcinoma (ccRCC) belongs to the TMB worse categories in $\mathrm{Wu}$ et al. study. High TMB in ccRCC has been reported by Zhang et al. to be associated with poor survival outcomes, advanced pathologic stage and higher tumor grade. Interestingly, this study also found lower immune cell infiltrates including (CD8+ T cell, CD4+ memory resting T cell, M1 and M2 macrophages) in TMB high group, which were associated with mutants of 6 hub TMB-related immune signature (11). This might conflict with findings in other studies, which reported that renal cell carcinoma including clear cell, carry the highest IDB amongst solid tumors, which might predict a higher potential for generating immunogenic peptides that substantiate the anti-tumor immune response, and thus response to immunotherapy (12).

Multiple clinical trials assessed the role of TMB as a predictive biomarker for response to immunotherapy. The CheckMate 227 trial (NCT02477826) demonstrated that high TMB in NSCLC $(\geq 10 \mathrm{mut} / \mathrm{Mb})$ is associated with a longer progression-free survival in nivolumab plus ipilimumab versus chemotherapy regardless of PD-L1 
expression (13). In addition, multiple other studies also correlated high TMB in variety of tumor types including NSCLC, urothelial carcinoma, cutaneous melanoma and colorectal cancer with the response to PD-1/PD-L1 blockade $(9,14)$.

Based on the findings from $\mathrm{Wu}$ et al. study, we question how TMB prognostic categories would be reflective in predicting response to immunotherapy. TMB as a predictive biomarker for immunotherapy on its own might not be as efficient as being predictive of prognosis. The amount of mutations a tumor has is not a mirror image of the degree of immunogenicity of created neoantigens. A good example of this is the case of Merkel cell carcinoma (9), a tumor type with a very low mutation burden. It is well known that this tumor has causative association with viral infection which impact mRNA editing and leads to creation of potential immunogenic peptides, and thus these tumors are responsive to immunotherapy.

As highlighted earlier, IDB, though small in its impact on prognosis might be more powerful than TMB in predicting the neoantigen burden, based on the fact that INDEL mutations have high probability of creating stronger immunogenic peptides then SNVs, and thus might be more useful as a biomarker for response to immunotherapy. Predicting the neoantigen burden in addition to the underlying specific molecular and immune genetic signatures might be the next feasible step to assess in the context of TMB and IDB prognostic categories in order to dissect where TMB and IDB stands in this realm.

There are lots of questions yet to be addressed in future prospective clinical trials. Such trials should be based on a multivariable model that would further validate TMB and IDB as biomarkers for therapy or prognosis in association with underlying molecular and immune genetic signatures specific to each tumor subtype. Findings from those studies will hopefully pave the way to better personalized immuneoncology approaches.

\section{Acknowledgments}

Funding: None.

\section{Footnote}

Conflicts of Interest: Both authors have completed the ICMJE uniform disclosure form (available at http://dx.doi. org/10.21037/atm-2020-75). AS reports grants and personal fees from AstraZeneca, grants and personal fees from
Bristol Myers Squibb, grants and personal fees from Merck, grants and personal fees from Exelixis, grants from Clovis, outside the submitted work. The other author has no other conflicts of interest to declare.

Ethical Statement: The authors are accountable for all aspects of the work in ensuring that questions related to the accuracy or integrity of any part of the work are appropriately investigated and resolved.

Open Access Statement: This is an Open Access article distributed in accordance with the Creative Commons Attribution-NonCommercial-NoDerivs 4.0 International License (CC BY-NC-ND 4.0), which permits the noncommercial replication and distribution of the article with the strict proviso that no changes or edits are made and the original work is properly cited (including links to both the formal publication through the relevant DOI and the license). See: https://creativecommons.org/licenses/by-nc-nd/4.0/.

\section{References}

1. Schumacher TN, Schreiber RD. Neoantigens in cancer immunotherapy. Science 2015;348:69-74.

2. Jiang T, Shi T, Zhang H, et al. Tumor neoantigens: from basic research to clinical applications. J Hematol Oncol 2019;12:93.

3. Meléndez B, Van Campenhout C, Rorive S, et al. Methods of measurement for tumor mutational burden in tumor tissue. Transl Lung Cancer Res 2018;7:661-7.

4. Yarchoan M, Hopkins A, Jaffee EM. Tumor mutational burden and response rate to PD-1 inhibition. $\mathrm{N}$ Engl J Med 2017;377:2500-1.

5. Vogelstein B, Papadopoulos N, Velculescu VE, et al. Cancer genome landscapes. Science 2013;339:1546-58.

6. Smith CC, Selitsky SR, Chai S, et al. Alternative tumourspecific antigens. Nat Rev Cancer 2019;19:465-78.

7. Wu HX, Wang ZX, Zhao Q, et al. Tumor mutational and indel burden: a systematic pan-cancer evaluation as prognostic biomarkers. Ann Transl Med 2019;7:640.

8. Devarakonda S, Rotolo F, Tsao MS, et al. Tumor mutation burden as a biomarker in resected non-small-cell lung cancer. J Clin Oncol 2018;36:2995-3006.

9. Goodman AM, Kato S, Bazhenova L, et al. Tumor mutational burden as an independent predictor of response to immunotherapy in diverse cancers. Mol Cancer Ther 2017; 16:2598-608.

10. Strickland KC, Howitt BE, Shukla SA, et al. Association 


\section{Page 4 of 4}

and prognostic significance of BRCA1/2-mutation status with neoantigen load, number of tumor-infiltrating lymphocytes and expression of PD-1/PD-L1 in high grade serous ovarian cancer. Oncotarget 2016;7:13587-98.

11. Zhang C, Li Z, Qi F, et al. Exploration of the relationships between tumor mutation burden with immune infiltrates in clear cell renal cell carcinoma. Ann Transl Med 2019;7:648.

12. Turajlic S, Litchfield K, Xu H, et al. Insertion-anddeletion-derived tumour-specific neoantigens and the immunogenic phenotype: a pan-cancer analysis. Lancet

Cite this article as: Saeed A, Salem ME. Prognostic value of tumor mutation burden (TMB) and INDEL burden (IDB) in cancer: current view and clinical applications. Ann Transl Med 2020;8(9):575. doi: 10.21037/atm-2020-75

\section{Saeed and Salem. Current view of tumor mutation and IDB in cancer}

Oncol 2017;18:1009-21.

13. Hellmann MD, Ciuleanu TE, Pluzanski A, et al. Nivolumab plus ipilimumab in lung cancer with a high tumor mutational burden. N Engl J Med 2018;378:2093-104.

14. Rizvi H, Sanchez-Vega F, La K, et al. Molecular determinants of response to anti-programmed cell death (PD)-1 and anti-programmed death-ligand 1 (PD-L1) blockade in patients with non-small-cell lung cancer profiled with targeted next-generation sequencing. J Clin Oncol 2018;36:633-41. 